\title{
A derivation of the Transient Statistical Energy Analysis (TSEA) equations with benchmark applications to plate systems
}

\author{
R.S. Langley ${ }^{a}$, D.H. Hawes*a, T. Butlin ${ }^{a}$, Y. Ishiib

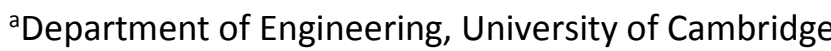 \\ Trumpington Street, Cambridge, CB2 1PZ, UK \\ ${ }^{b}$ Mitsubishi Heavy Industries, Ltd. 5-717-1, \\ Fukahori-machi, Nagasaki, 851-0392, Japan
}

* Corresponding author

Email address: David.HAWES@3ds.com

Present address: Dassault Systèmes, Cambridge Science Park, Cambridge, CB4 OWN, UK

\begin{abstract}
Statistical Energy Analysis (SEA) is a well-established method for predicting the response of complex systems to high frequency steady-state harmonic or random excitation. The method has also been applied to transient and shock loading, in which case it is referred to as Transient SEA (TSEA), although the validity of the approach is less certain for this case since the TSEA equations mix time and frequency descriptions of the response in an ad-hoc way. In this paper, the TSEA equations are derived in a new way by employing an analogy of the Priestley description of a non-stationary random process. A key feature is that shock loading is deterministic, so that the random ensemble of responses arises from random structural properties rather than random loading, and this requires a reinterpretation of the Priestley description. The present derivation of TSEA enables the appropriate initial conditions
\end{abstract}


on the equations to be established and bounds on the prediction error are found from Parseval's theorem. The derived equations are applied to numerical and experimental examples involving plates.

Keywords: TSEA; Transient vibration; Shock-induced vibration; Priestley description

\section{Introduction}

The development of Statistical Energy Analysis (SEA) arose from demand for efficient vibration modelling at high frequencies where the limitations of conventional vibration modelling techniques impede their applicability. At these frequencies, manufacturing imperfections in the structure are of the lengthscale of the vibration wavelengths and can therefore have a significant influence on the response meaning analysis of an ensemble of systems is required. Additionally, for elemental methods the length scales involved require an extremely detailed model. For both of these reasons, a conventional deterministic approach becomes extremely computationally expensive and SEA, which accounts for these issues, becomes attractive.

In many applications, a structure will be excited by a steady load with properties that do not vary with time, for example a car travelling at a fixed speed will experience steady engine and aerodynamic forces. Consequently, a substantial body of research exists to model the response of systems under these forms of loading. Statistical Energy Analysis is a prominent method in which a system is divided up into regions known as "subsystems" and the vibrational energy of each subsystem is calculated, see for example [1]. The resulting SEA equation, derived by Lyon [1] by considering the power balance within a subsystem can be solved at a given excitation frequency, $\omega$, to predict the total energy in each subsystem.

In general, however, structures will not only be subject to steady loading conditions, but also impulsive and time-varying forces that can cause high stresses and undesirable noise. The limitations of conventional vibration modelling approaches are the same at high frequencies for transient loading leading to many authors investigating the extension of SEA to the transient case, notably [1-6]. However, in contrast to steady-state loading there is no rigorously derived, generally recognised method for transient Statistical Energy Analysis (TSEA). Nonetheless, consistent across all approaches is the addition of the time derivative of energy to the SEA equation such that the power balance accounts for build-up or loss of energy over time in each subsystem. 
Whilst extension to the TSEA equation may seem reasonable, a number of issues arise:

1. Is the addition of an energy time derivative term physically justifiable?

2. Are the transient loss factors the same as those used in steady-state analysis?

3. The frequency and time domains have been mixed in a non-rigorous way.

4. What initial condition should be used for the energy variable under impulsive loading?

Provided the assumptions applied in the steady-state derivation of SEA remain valid, the TSEA equation should be a legitimate extension. This requires the energy variables and power input to vary significantly slower than the oscillations of the system and many vibration modes to be excited, a condition that is met under impulsive loading. Under these conditions, the loss factors required will be unlikely to vary significantly from the steady-state loss factors, despite the conclusion in [5] that time varying loss factors are more appropriate, particularly at low frequencies. Nevertheless, in this paper the same loss factors as those used for a steady-state analysis are used since they have been found to produce good results here, as well as in [7] where the issue is also discussed, and to preserve the simplicity of TSEA. The remaining issues are the subject of this paper; a formal approach to describing the time varying energy is developed by combining the Stieltjes integral with the nonstationary random vibration description of Priestley $[8,9]$ and a general method for calculating the initial energy after an impulse is provided, similar to [3], via use of Parseval's theorem.

A number of authors have previously investigated the TSEA equation, although often the energy variable is treated as the energy in a frequency band rather than a function of both time and frequency and mostly systems with only two subsystems are investigated. Mixed results have been published $[1-7,10-12]$, with good accuracy in some cases and poorer results in others, although this may be due to the system or frequency range selected and because only a single realisation rather than ensemble of systems was investigated. In this paper, strong results are produced for TSEA provided the system produces accurate steady-state SEA results.

It is shown in [3] that Parseval's theorem can be used to relate the area under the energy curve in the time and frequency domains and, in this paper, this approach is used to calculate the initial energy in a impulsively excited subsystem. Methods for calculating the input power from impulsive loading using experimental or numerical data or the subsystem impedance are discussed in [11] and in [13] SEA results are used to estimate the location and waveform of an impulsive input using Parseval's theorem.

A known deficiency of TSEA due to its diffusive nature is that it cannot predict localisation effects as described in $[14,15]$ whereby even at large times, energy remains localised in a subsystem and 
equipartition does not occur. This arises in systems with low modal density, weak coupling and very low damping where the time required for energy to diffuse through a system is longer than the timescale of actual energy transport. The equivalent limitation for steady-state SEA has been investigated in [16] which demonstrates that for a system with strong localisation, the coupling loss factors should be proportional to the damping. This issue is not considered in this paper because realistic damping levels override its effects [14] and since the accuracy of steady-state and transient SEA are related, it is not solely a deficiency of TSEA and is therefore outside the scope of this paper.

Another issue with TSEA is that it assumes infinitely fast energy transfer between excited and distant subsystems. For example, consider a number of subsystems coupled in series, with an impulse applied to the first subsystem. After an infinitesimal time, the TSEA solution will yield a finite, albeit small, energy in the furthest subsystem, when in reality, energy will take time to travel out from the excitation point and cascade through the subsystems. In order to account for this effect, it has been suggested $[7,11,17]$ that numerical integration of the TSEA equation with a minimum timestep, related to the time taken for energy to diffuse within a subsystem, should be used to provide a solution. This issue occurs when the timescale for energy to travel across a subsystem and therefore a reverberant field to build up is significant compared to the timescale for the energy in a subsystem to become a maximum following an impulse in a neighbouring subsystem. The relationship between these two timescales depends strongly on both the damping in the system and the coupling strength and is favourable for light damping and weak coupling, two criteria required for an accurate SEA application. In a highly damped case, the energy in an impulsively excited subsystem dissipates rapidly and TSEA can predict a large proportion of the overall energy transfer occurs immediately after the impulse, possibly before the outgoing wave from an impulse has even reached the coupling. Similarly, when the coupling is strong TSEA predicts rapid energy transfer instantaneously after an impulse, again, possibly before the outgoing wave from an impulse has even reached the coupling. However, when damping is light and coupling is weak, a reverberant field will build up in a subsystem before significant loss to internal damping or other subsystems thus the exact TSEA solution should be appropriate. In this paper, the accuracy of steady-state and transient SEA predictions are shown to be strongly related and so the deficiencies discussed above for TSEA have equivalent deficiencies under steady-state conditions. Any system demanding a non-exact TSEA solution will therefore also exhibit poor steadystate SEA results. Consequently, modifying the timestep risks applying an SEA approach in conditions where it is inherently inaccurate.

In what follows, a rigorous definition of the energy variable of the TSEA equation is derived using the Priestley description in Section 2 before a general method for solving the equation and applying 
realistic initial conditions is developed in Section 3. Validation of this method using numerical simulations and experiment results is then presented in Sections 4 and 5 respectively.

\section{The Priestley Description}

Transient SEA combines the time and frequency domains to predict the time-varying subsystem energies of a single frequency or a frequency band. To model this, a rigorous definition of the energy variable must be provided and the formalism introduced by Priestley $[8,9]$ to study non-stationary random vibration can be applied. However, in this case instead of the randomness arising from the input, it arises from the randomness across the ensemble of systems as justified in the Appendix. A general stationary random variable $v(t)$ can be related to its Fourier transform via the expression

$$
v(t)=\int_{-\infty}^{\infty} V(\omega) \mathrm{e}^{\mathrm{i} \omega t} \mathrm{~d} \omega
$$

and the mean squared value of the variable then has form

$$
\mathrm{E}\left[v^{2}(t)\right]=\int_{-\infty}^{\infty} \int_{-\infty}^{\infty} \mathrm{E}\left[V(\omega) V^{*}\left(\omega^{\prime}\right)\right] \mathrm{e}^{\mathrm{i}\left(\omega-\omega^{\prime}\right) t} \mathrm{~d} \omega \mathrm{d} \omega^{\prime}
$$

where $E[.$.$] represents the ensemble average with the ensemble consisting of the selection of random$ systems. In order for Eq. (2) to be compatible with the single-sided spectrum $S_{v v}(\omega)$ of $v(t)$ we must have

$$
\mathrm{E}\left[V(\omega) V^{*}\left(\omega^{\prime}\right)\right]=\frac{1}{2} S_{v v}(\omega) \delta\left(\omega-\omega^{\prime}\right), \quad \mathrm{E}\left[v^{2}(t)\right]=\int_{0}^{\infty} S_{v v}(\omega) \mathrm{d} \omega .
$$

Equation (1) can also be expressed as a so-called Stieltjes integral in the form

$$
\begin{gathered}
v(t)=\int_{-\infty}^{\infty} \mathrm{e}^{\mathrm{i} \omega t} \mathrm{~d} S(\omega), \\
\mathrm{d} S(-\omega)=\mathrm{d} S^{*}(\omega), \quad \mathrm{E}\left[\mathrm{d} S(\omega) \mathrm{d} S^{*}\left(\omega^{\prime}\right)\right]=\frac{1}{2} S_{v v}(\omega) \delta\left(\omega-\omega^{\prime}\right) \mathrm{d} \omega \mathrm{d} \omega^{\prime} .
\end{gathered}
$$

Equation (5) forms the basis of Priestley's representation of a non-stationary random process, in which the equation is generalized to

$$
v(t)=\int_{-\infty}^{\infty} V(\omega, t) \mathrm{e}^{\mathrm{i} \omega t} \mathrm{~d} S(\omega),
$$

where $V(\omega, t)$ is a deterministic function with

$$
V(-\omega, t)=V^{*}(\omega, t) .
$$

The mean squared value of $v(t)$ is then time varying with

$$
\mathrm{E}\left[v^{2}(t)\right]=\int_{0}^{\infty}|V(\omega, t)|^{2} S_{v v}(\omega) \mathrm{d} \omega .
$$

Note that without loss of generality in the Priestley description we can put 


$$
S_{v v}(\omega)=1 \text {, }
$$

so that $V(\omega, t)$ acts on white noise. If $v(t)$ represents the velocity at a point in subsystem $j$ then by taking the spatial average of $v^{2}(t)$ over the subsystem, we can identify the energy of the $j$ th subsystem, $E_{j}(\omega, t)$, as being entirely analogous to $|V(\omega, t)|^{2}$ such that

$$
E_{j}(\omega, t)=M_{j}<|V(\omega, t)|^{2}>
$$

where $M_{j}$ is the mass of the subsystem, and <..> represents the spatial average. The time evolution of the total energy can be found using Eq. (10) as

$$
E_{\mathrm{tot}, j}(t)=\int_{0}^{\infty} E_{j}(\omega, t) \mathrm{d} \omega .
$$

In these equations, $E_{j}(\omega, t)$ is the evolutionary spectrum of the energy in subsystem $j$ and has been found by supposing that the velocity of a point in the subsystem takes the form of Eq. (8), where dS has the properties defined in Eqs. (6) and (7) and then spatially averaging over the square of the velocity. If a deterministic analysis was required and the local velocity was of interest, Eqs. (7) and (8) could provide the response; however, SEA is interested more simply in the ensemble average of the subsystem energy and so only $E_{j}(\omega, t)$ is required.

This description of the subsystem energies will form the basis of the TSEA method which can be found by considering a transient power balance for subsystem $j$ such that

$$
P_{\mathrm{in}, j}(\omega, t)=\frac{\partial E_{j}(\omega, t)}{\partial t}+P_{\mathrm{diss}, j}(\omega, t)+P_{\mathrm{trans}, j}(\omega, t)
$$

where $P_{\mathrm{in}, j}(\omega, t), P_{\mathrm{diss}, j}(\omega, t)$ and $P_{\mathrm{trans}, j}(\omega, t)$ are the evolutionary spectra of the external power input into, power dissipated by, and power transferred into other subsystems from the $j$ th subsystem. If the system is assumed to satisfy the standard steady-state SEA assumptions [1] then the rightmost two power terms can be combined to yield the transient SEA equation in matrix form

$$
\mathbf{P}_{\text {in }}(\omega, t)=\frac{\partial \mathbf{E}(\omega, t)}{\partial t}+\mathbf{A}(\omega) \mathbf{E}(\omega, t)
$$

or using index notation

$$
P_{\mathrm{in}, j}=\frac{\partial E_{j}}{\partial t}+\omega \eta_{j} E_{j}+\sum_{k=1}^{N} \omega \eta_{j k} n_{j}\left(\frac{E_{j}}{n_{j}}-\frac{E_{k}}{n_{k}}\right)
$$

where $n_{j}$ and $\eta_{j}$ are the modal density and loss factor of subsystem $j$ and $\eta_{j k}$ is the coupling loss factor between subsystems $j$ and $k$ and the arguments have been removed for brevity. It should be noted that Eq. (14) is exact, but since the SEA assumptions have been applied, Eq. (15) is an approximation and consequently the SEA matrix, $\mathbf{A}(\omega)$, is time-invariant. 


\section{Solution of the TSEA equations}

Using the Priestley notation, the TSEA equation, Eq. (15), can be investigated. For a general power input, the solution to the TSEA equation will consist of the complementary function and particular integral and so can be written in the form

$$
\mathbf{E}(\omega, t)=\exp (-\mathbf{A}(\omega) t)\{\mathbf{E}(\omega, 0)+\mathbf{Z}(\omega, t)\},
$$

where $\mathbf{Z}(\omega, 0)=\mathbf{0}$. Equation (17) can be substituted into Eq. (15) to provide

$$
\frac{\mathrm{d} \mathbf{Z}(\omega, t)}{\mathrm{d} t}=\exp (\mathbf{A}(\omega) t) \mathbf{P}_{\mathrm{in}}(\omega, t)
$$

and it follows that

$$
\mathbf{Z}(\omega, t)=\int_{0}^{t} \exp (\mathbf{A}(\omega) \tau) \mathbf{P}_{\text {in }}(\omega, \tau) \mathrm{d} \tau
$$

Substituting Eq. (19) into Eq. (17) then yields the general solution of the TSEA equation

$$
\mathbf{E}(\omega, t)=\exp (-\mathbf{A}(\omega) t) \mathbf{E}(\omega, 0)+\int_{0}^{t} \exp (-\mathbf{A}(\omega)(t-\tau)) \mathbf{P}_{\text {in }}(\omega, \tau) \mathrm{d} \tau
$$

Since the SEA equation has been derived for systems in steady-state, for this equation to provide a reasonable calculation of the subsystem energies, the power input and subsystem energies must vary slowly with time when compared to the oscillation of the system.

In this section, only shock loads will be considered and it is assumed that the time for the power to be input to the system by a shock load is much shorter than the reverberation time of the system so that the power input can be considered to be instantaneous. The loading can therefore be applied to Eq. (20) as an initial condition rather than an external power input.

An efficient solution to Eq. (15) can be obtained by computing the eigenvalues and eigenvectors of the matrix $\mathbf{A}$. If $\boldsymbol{\Phi}$ is a matrix whose columns are the eigenvectors of $\mathbf{A}$ and $\boldsymbol{\Lambda}$ is a diagonal matrix with diagonal entries given by the eigenvalues of $\mathbf{A}$, the solution becomes

$$
\mathbf{E}(\omega, t)=\boldsymbol{\Phi} \exp (-\boldsymbol{\Lambda} t) \boldsymbol{\Phi}^{-1} \mathbf{E}(\omega, 0) .
$$

For the transient solution given by Eq. (21), all that is required is an appropriate initial condition, $\mathbf{E}(\omega, 0)$, to be applied. This can be explored by integrating Eq. (21) over time to yield

$$
\int_{0}^{\infty} \mathbf{E}(\omega, t) \mathrm{d} t=\boldsymbol{\Phi} \int_{0}^{\infty} \exp (-\boldsymbol{\Lambda} t) \mathrm{d} t \boldsymbol{\Phi}^{-1} \mathbf{E}(\omega, 0)=\left\{\boldsymbol{\Phi} \boldsymbol{\Lambda}^{-1} \boldsymbol{\Phi}^{-1}\right\} \mathbf{E}(\omega, 0)=\mathbf{A}^{-1}(\omega) \mathbf{E}(\omega, 0)
$$

and then integrating over both frequency and time to give 


$$
\int_{0}^{\infty} \mathbf{E}_{\mathrm{tot}}(t) \mathrm{d} t=\int_{0}^{\infty} \int_{0}^{\infty} \mathbf{E}(\omega, t) \mathrm{d} t \mathrm{~d} \omega=\int_{0}^{\infty} \mathbf{A}^{-1}(\omega) \mathbf{E}(\omega, 0) \mathrm{d} \omega=\int_{0}^{\infty} \mathbf{E}_{\mathrm{ss}}(\omega) \mathrm{d} \omega,
$$

where $\mathbf{E}_{\mathrm{ss}}(\omega)$ is defined such that

$$
\mathbf{A E}_{\mathrm{ss}}=\mathbf{E}(\omega, 0)
$$

thus $\mathbf{E}_{\mathrm{SS}}(\omega)$ is the steady-state SEA solution to a power input of $\mathbf{E}(\omega, 0)$.

The initial condition $\mathbf{E}(\omega, 0)$ should be chosen so that Eq. (23) is a meaningful and correct equation, and this can be achieved by interpreting Eq. (23) as a version of Parseval's theorem, which states

$$
\int_{0}^{\infty} v_{j}^{2}(t) \mathrm{d} t=2 \pi \int_{-\infty}^{\infty}\left|v_{j}(\mathrm{i} \omega)\right|^{2} \mathrm{~d} \omega=4 \pi \int_{0}^{\infty}\left|v_{j}(\mathrm{i} \omega)\right|^{2} \mathrm{~d} \omega,
$$

for any function $v_{j}(t)$ (taken here to be zero for $t<0$ ) and its Fourier transform $v_{j}(i \omega)$. If $v_{j}(t)$ is interpreted as a velocity in subsystem $j$ caused by an impulsive force $f(t)$ applied to subsystem $k$, and we write

$$
\begin{gathered}
E_{\mathrm{tot}, j}(t)=M_{j}<v_{j}^{2}(t)>, \\
E_{\mathrm{ss}, j}(\omega)=4 \pi M_{j}<\left|v_{j}(\mathrm{i} \omega)\right|^{2}>,
\end{gathered}
$$

then Eq. (23) will be correct by virtue of Parseval's theorem. The value of the initial condition, $E_{k}(\omega, 0)$, should therefore be chosen such that $E_{\mathrm{ss}, j}(\omega)$ is given by Eq. (27).

The term $E_{k}(\omega, 0)$ in Eq. (24) can be interpreted as the power input by a harmonic force of complex amplitude $f(\mathrm{i} \omega)$, this being the Fourier transform of the impulsive force $f(t)$, and found by considering the modal density of the subsystem [18]. The solution of the steady-state SEA equations provides energy in the subsystems from this power input as

$$
E_{k}(\omega, 0)=\left(\frac{\pi n_{k}}{4 M_{k}}\right)|f(\mathrm{i} \omega)|^{2} \quad \rightarrow \quad E_{\mathrm{ss}, j}(\omega)=\left(\frac{M_{j}}{2}\right)<\left|v_{j}(\mathrm{i} \omega)\right|^{2}>
$$

It follows that to enforce Eq. (27), the equivalent input power should be multiplied by a factor of $8 \pi$ so that

$$
E_{k}(\omega, 0)=8 \pi\left(\frac{\pi n_{k}}{4 M_{k}}\right)|f(\mathrm{i} \omega)|^{2} \quad \rightarrow \quad E_{\mathrm{ss}, j}(\omega)=4 \pi M_{j}<\left|v_{j}(\mathrm{i} \omega)\right|^{2}>
$$

Applying this initial condition to Eq. (20) satisfies Parseval's theorem in Eq. (23), ensuring that the area under the transient energy curve will be correct, provided the area under the frequency curve for the equivalent steady-state SEA model is correct. 


\section{Numerical validation}

In this section, the TSEA theory of Section 3 for predicting the mean subsystem energies in an SEA system is validated via comparison with results calculated using the finite element method (FE). The commercial finite element package COMSOL was used to model plate structures and generate the stiffness and mass matrices required for subsequent modal analysis.

\subsection{Methodology}

In all cases investigated, an impulsive load is applied meaning that the external power input in Eq. (15) is set to zero. Since SEA is generally applied to energy at high frequencies, the impulse applied can be thought to only excite within a given frequency band. This can be applied using one of two approaches; either it can be modelled as exciting only the modes in the frequency range of interest or it can be modelled as a signal containing equal frequency input at all frequencies in the range of interest and zero input elsewhere. For a narrow frequency range, the modal technique provides poor results immediately after the impulse for subsystems that are not directly forced since there are insufficient modes in the band to adequately cancel when summed and produce zero energy far from the input.

Modelling the impulse as possessing a flat frequency response over the range of interest is also problematic for small frequency ranges. In the time domain, an impulse of this form is comprised of the subtraction of one sinc function from another with bandwidths provided by the upper and lower limits respectively of the frequency range of interest. For narrow frequency ranges, this provides a non-causal input, which differs from the input assumed by the TSEA theory. As the frequency range of interest widens, the two methods converge and since TSEA will in general be applied to a wide frequency band the discussed issues become insignificant. In the following simulations, the modal approach is adopted.

In order to generate an ensemble of structures to compare to SEA, the plates were randomised by adding to each plate five masses each of $2 \%$ and five masses each of $-2 \%$ of the plate mass and two springs of stiffness $(250 \times 2 \pi)^{2} M_{j}$ at random locations. The plate parameters used were for $5 \mathrm{~mm}$ thick Aluminium plate with Young's modulus $70 \mathrm{GPa}$, density $2700 \mathrm{~kg} \mathrm{~m}^{-3}$, Poisson ratio 0.33 , loss factor 0.03 and with all edges pinned to allow rotation, but zero displacement. Only out-of-plane modes were considered and an ensemble of 100 realisations over a frequency range of $500-1500 \mathrm{~Hz}$ was used. 


\subsection{Prediction of Mean Subsystem Energy}

For the system of Figure 1 consisting of two plates of areas 1.26 and $1.3 \mathrm{~m}^{2}$ coupled via three stiff point connectors, the mean transient and steady-state SEA results for plates one and two are compared to FE results in Figures 2 and 3 respectively. The TSEA results match the FE results very closely, with the initial energy in plate one and the rise and decay of energies in all plates being accurately predicted. Additionally, the time at which the peak occurs and its magnitude in plate two are very similar with a $9.3 \%$ overprediction in magnitude. The steady-state SEA is also shown to closely match the FE meaning that the area under the transient curves is very similar for SEA and FE. The direct relationship between the two is highlighted by the comparable overprediction of the steadystate and transient results for plate two. The non-zero initial energy in the unforced plate is a consequence of the summation of a finite number of modes being unable to cancel to zero velocity everywhere when superimposed.

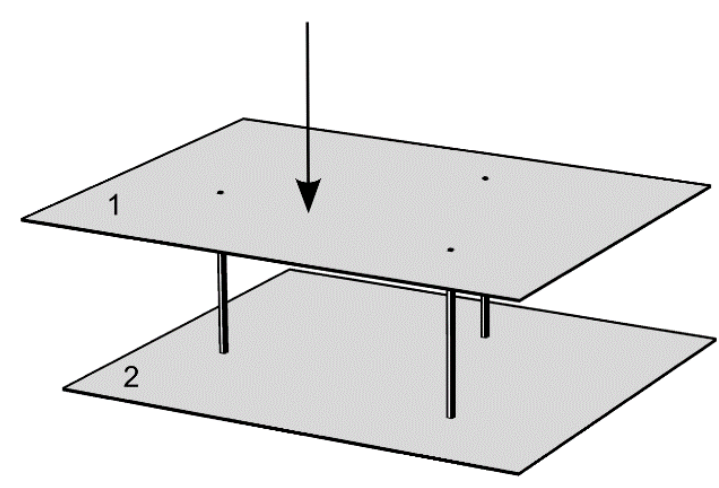

Figure 1: Two plate system with point couplings. 


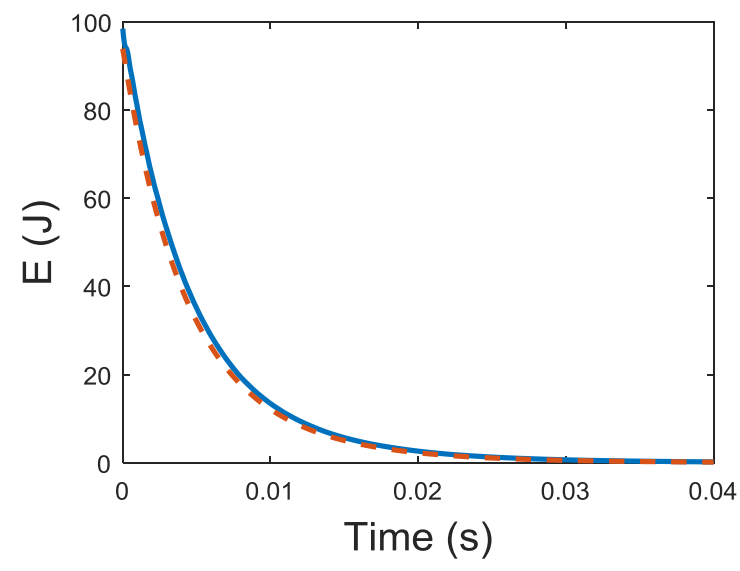

(a)

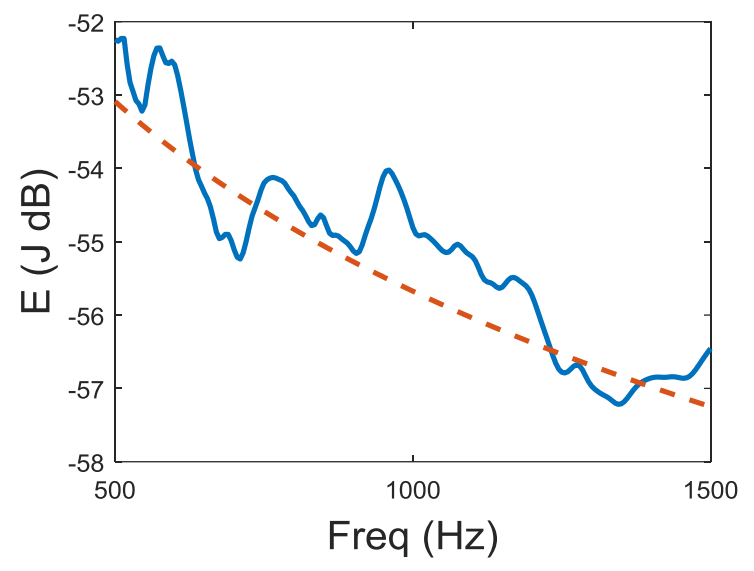

(b)

Figure 2: Mean energies predicted by SEA (dashed) compared with FE (solid) for plate one of the two plate system: (a) transient mean and (b) steady-state mean.

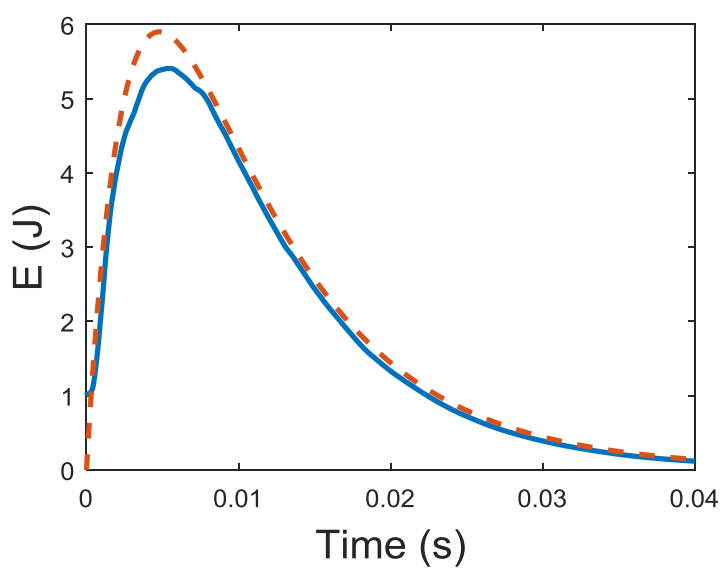

(a)

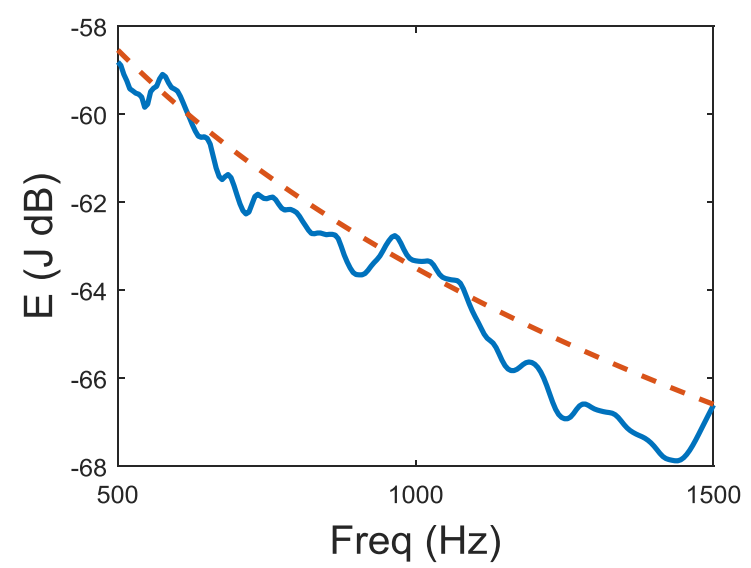

(b)

Figure 3: Mean energies predicted by SEA (dashed) compared with FE (solid) for plate two of the two plate system: (a) transient mean and (b) steady-state mean.

How to judge the accuracy of the transient results is an interesting question, since It is most common to plot steady-state SEA results on a logarithmic scale and it is generally accepted that errors of $\pm 3 \mathrm{~dB}$ are reasonable for steady-state SEA especially when considering the relative difference in subsystem energies can be orders of magnitude. When plotted on a linear scale, as in Figures 2 and 3 , a 3 dB error corresponds to approximately a factor of two and so the transient peak error for plate two of $9.3 \%$ is extremely positive. A more traditional comparison with the transient energy of both plates plotted on a logarithmic scale is displayed in Figure 4 where the relative difference in energy between the plates is emphasised meaning the small error in the TSEA prediction seems insignificant. The decay rate of the two subsystems is comparable to the FE results although, at late times the TSEA results tend to 
converge whereas the FE results do not. This is due to non-equipartition of energy through localisation as discussed in Section 1 and is of little practical significance here since the energy levels are so low compared to the peak levels.

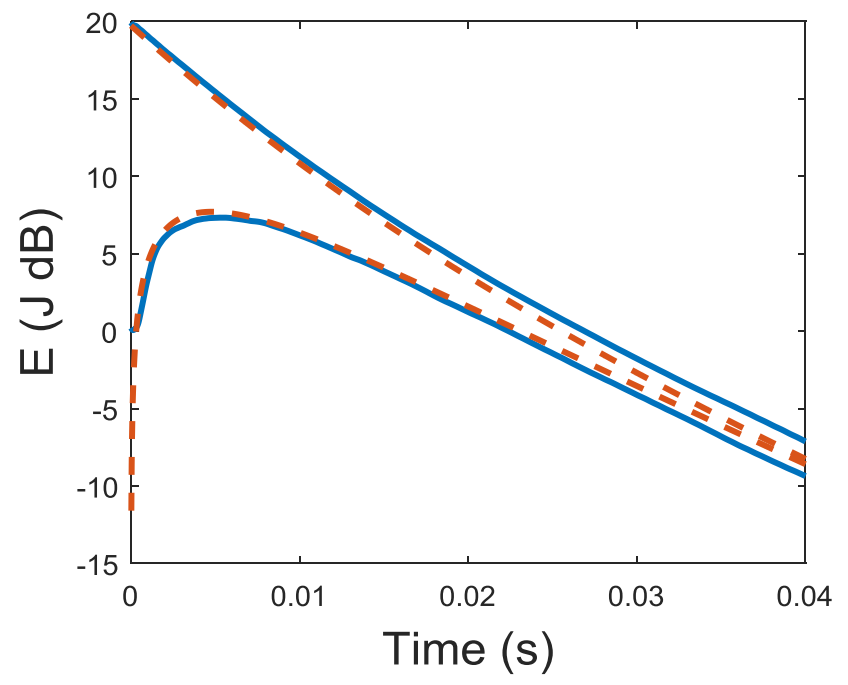

Figure 4: Mean energies predicted by SEA (dashed) compared with FE (solid) for both plates of the two plate system plotted on a logarithmic scale.

For the system of Figure 5 consisting of three plates of areas $1.62,1.26$ and $1.42 \mathrm{~m}^{2}$ coupled by $90-$ degree edge couplings, the steady-state and TSEA results for each subsystem are shown in Figures 68. As with the two plate system, the FE results are closely predicted by SEA, with both having similar rise and decays in mean energy. There are, however, slight differences between the TSEA and FE results, with TSEA underpredicting the peak energy by 8.0 and $13 \%$ in plates one and two and overpredicting by $18 \%$ in plate three. These differences can also be observed in the steady-state SEA, demonstrating that Parseval's theorem, Eq. (23), holds.

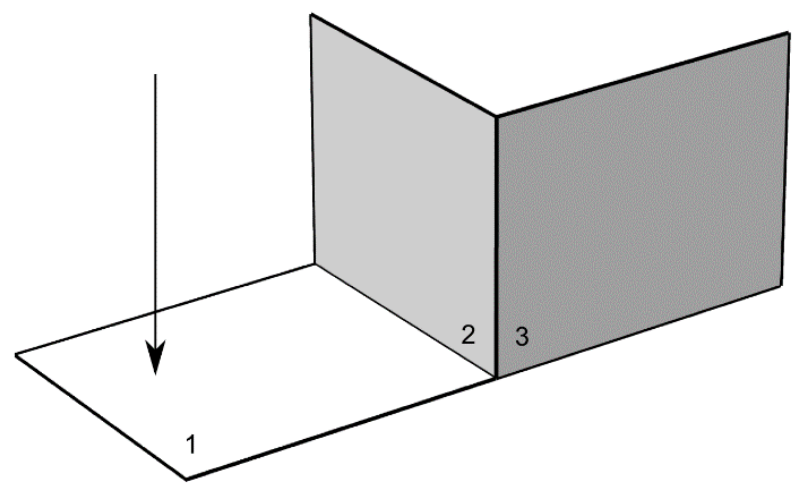

Figure 5: Three plate system with edge coupling. 


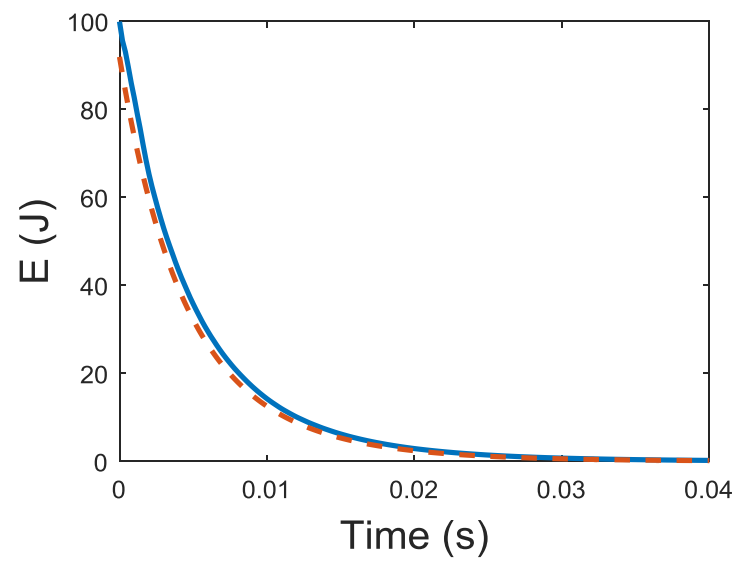

(a)

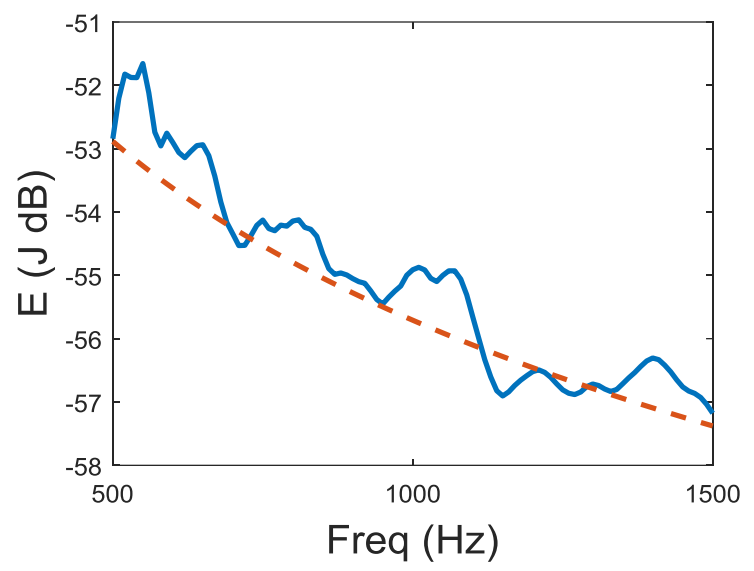

(b)

Figure 6: Mean energies predicted by SEA (dashed) compared with FE (solid) for plate one of the three plate system: (a) transient mean and (b) steady-state mean.

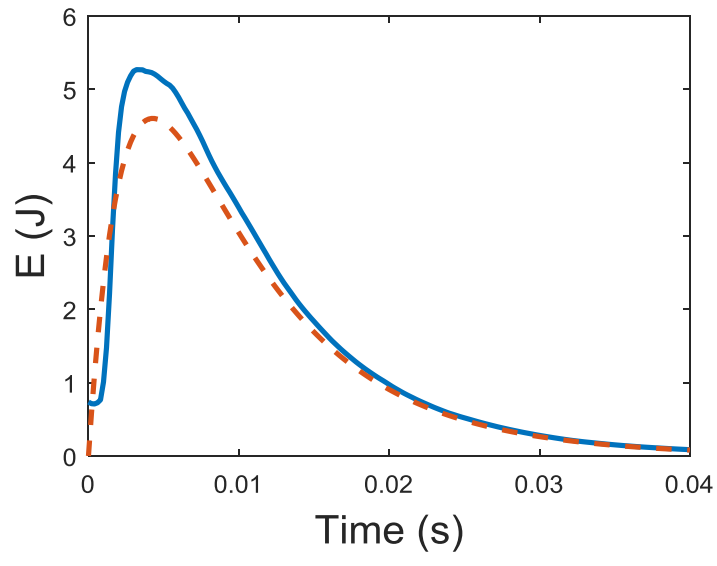

(a)

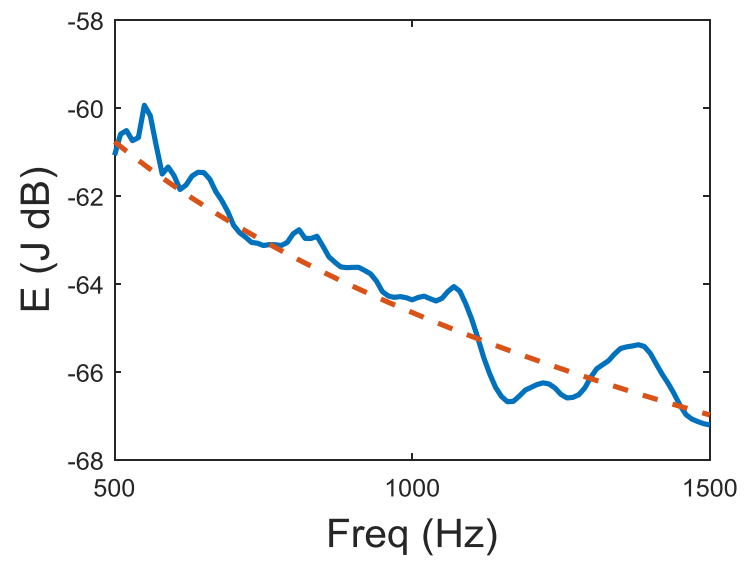

(b)

Figure 7: Mean energies predicted by SEA (dashed) compared with FE (solid) for plate two of the three plate system: (a) transient mean and (b) steady-state mean. 


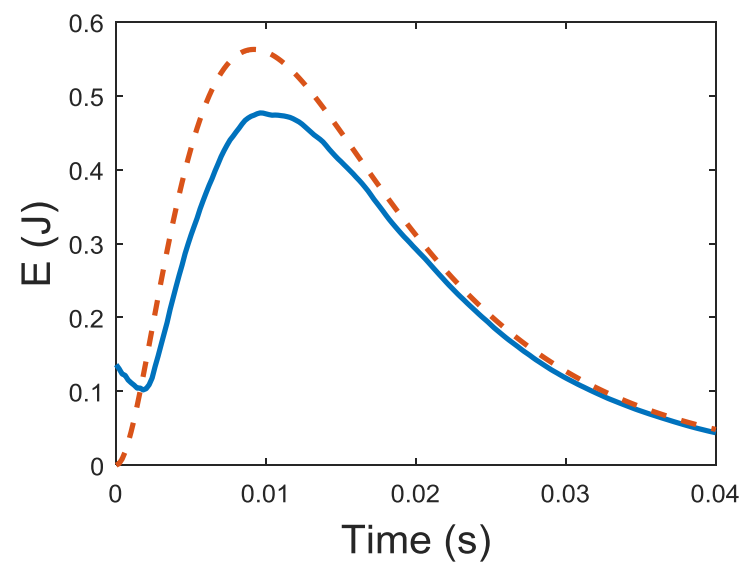

(a)

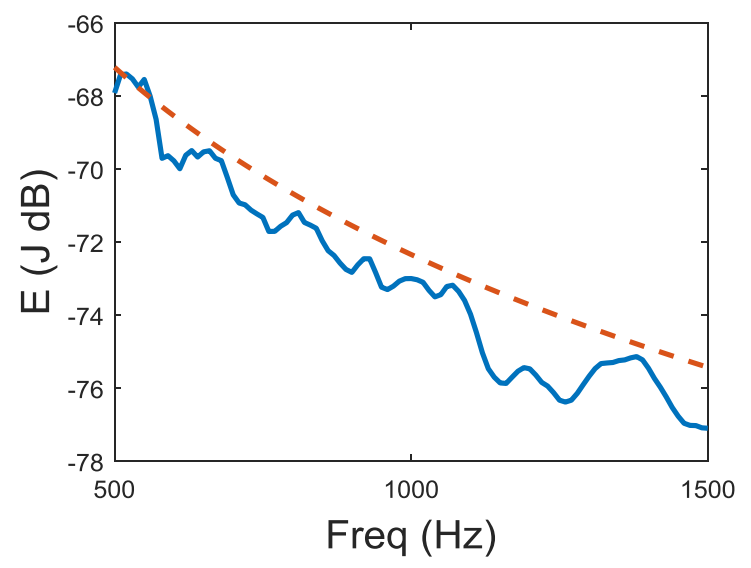

(b)

Figure 8: Mean energies predicted by SEA (dashed) compared with FE (solid) for plate three of the three plate system: (a) transient mean and (b) steady-state mean.

In order to isolate errors due to TSEA alone rather than those caused by inaccuracies in the application of steady-state SEA to this system, the coupling loss factors have been scaled to enforce equal areas under the steady-state SEA and FE curves for plate three. The areas under the TSEA and FE mean transient energy curves are now also equal due to Eq. (23) and Figure 9 shows that the TSEA results now match the FE results closely. Here, the peak predicted by the TSEA is within $2.5 \%$ of the peak from the FE. The rise time for the TSEA curve is shorter than the benchmark results, most likely due to the more detailed differences between the SEA and FE frequency responses.

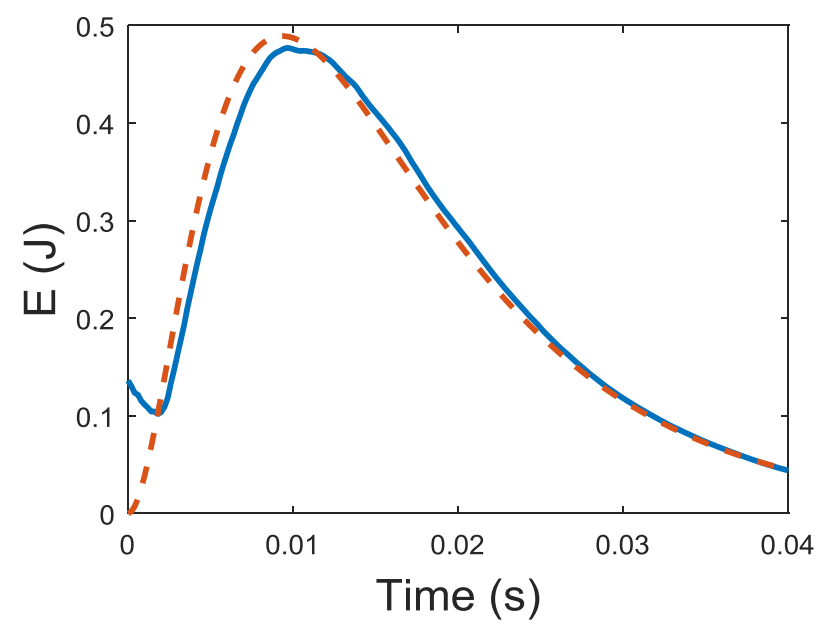

Figure 9: Mean energies predicted by SEA with modified coupling loss factors (dashed) compared with FE (solid) for plate three of the three plate system. 


\section{Experimental Validation}

To supplement the numerical validation of the TSEA method in Section 4, an experiment involving a two plate system similar to Figure 1 has also been conducted. Two aluminium plates with area 0.8 and $0.48 \mathrm{~m}^{2}$ were suspended using string and coupled with two steel point connections as displayed in Figure 10. An impulse was applied to the upper plate, denoted plate one, and the responses of the plates were measured using five randomly spaced accelerometers on each plate from which the average velocity and therefore an estimate of the energy of each plate was calculated. In order to randomise the modeshapes and generate an ensemble of systems, a number of masses, totalling approximately $10 \%$ of the plate mass, have been attached to each plate and are redistributed for each impulsive excitation.

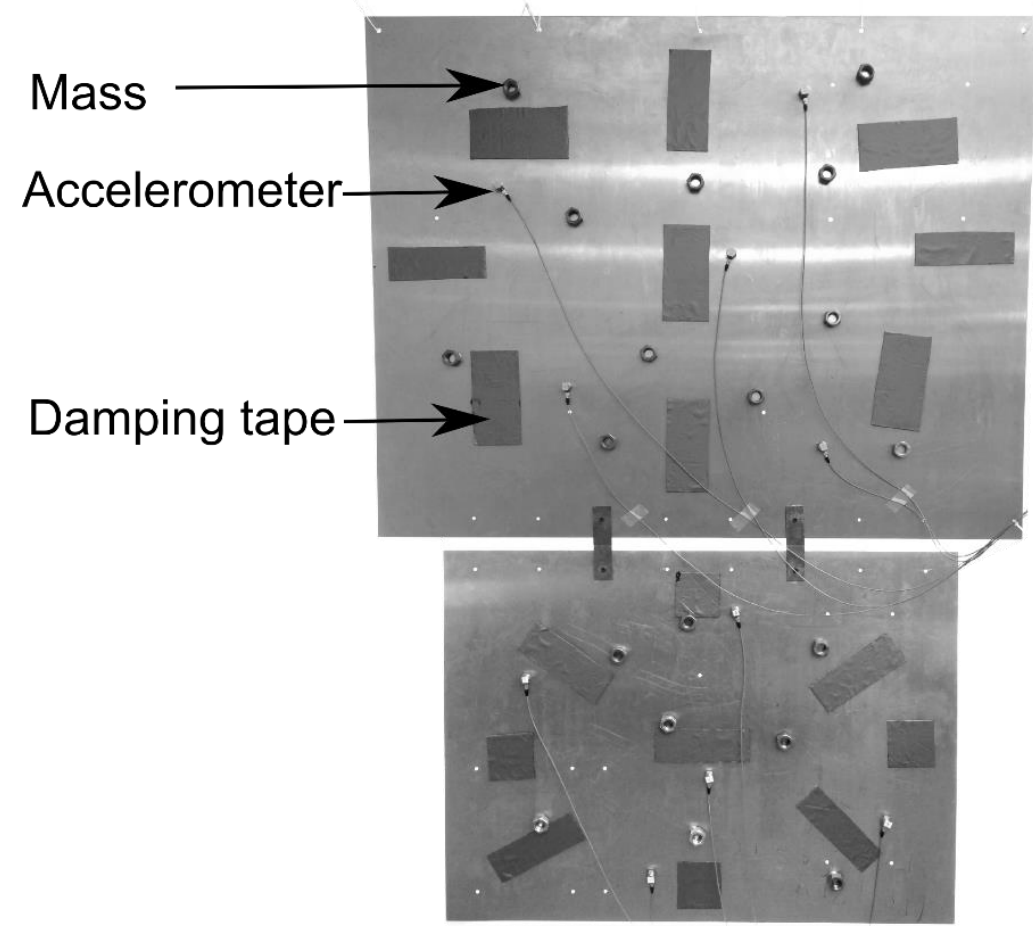

Figure 10: Experimental apparatus

The loss factors of each subsystem have been determined at a number of frequencies by investigating the decay curves of the subsystems in isolation and were found to be approximately 0.01 . The SEA coupling loss factors were then calculated from the experimental steady-state energy difference between each plate using

$$
\eta_{12}=\frac{n_{2} E_{2}}{n_{2} E_{1}-n_{1} E_{2}} \eta_{2}
$$


where the modal densities are calculated analytically for a plate and $\eta_{21}$ can be calculated using the reciprocity relationship $n_{1} \eta_{12}=n_{2} \eta_{21}$. The modal overlap factors at $1000 \mathrm{~Hz}$ are 1.25 and 0.75 for plates one and two respectively.

The experimental results are compared in Figures 11 and 12 to transient and steady-state SEA results in the frequency range $1000-2000 \mathrm{~Hz}$ where 10 realisations have been averaged and the data has been normalised such that the peak force of each impulse is $1 \mathrm{~N}$. As with the FE results of Section 4, strong agreement is seen between the experiment and TSEA and the accuracy of the TSEA is governed via Parseval's theorem by the accuracy of the steady-state SEA results. The TSEA can appear worse than its steady-state equivalent, but this is a result of observing transient results on a linear scale and steady-state results on a logarithmic scale.

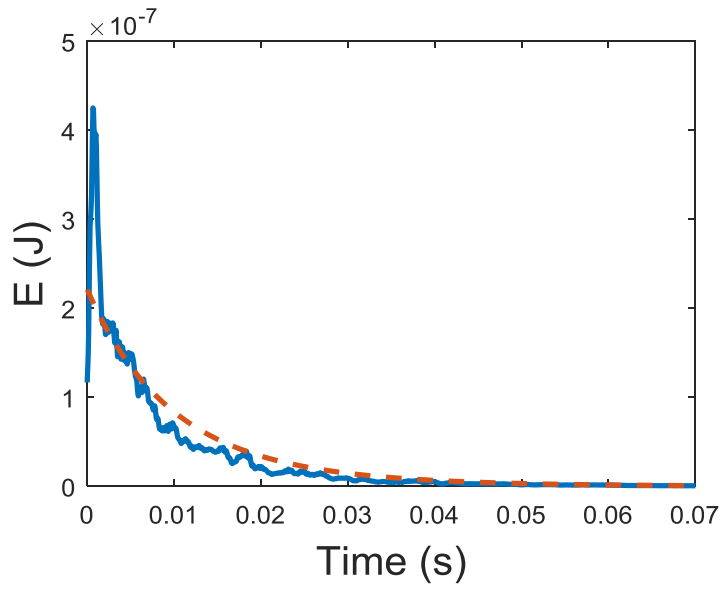

(a)

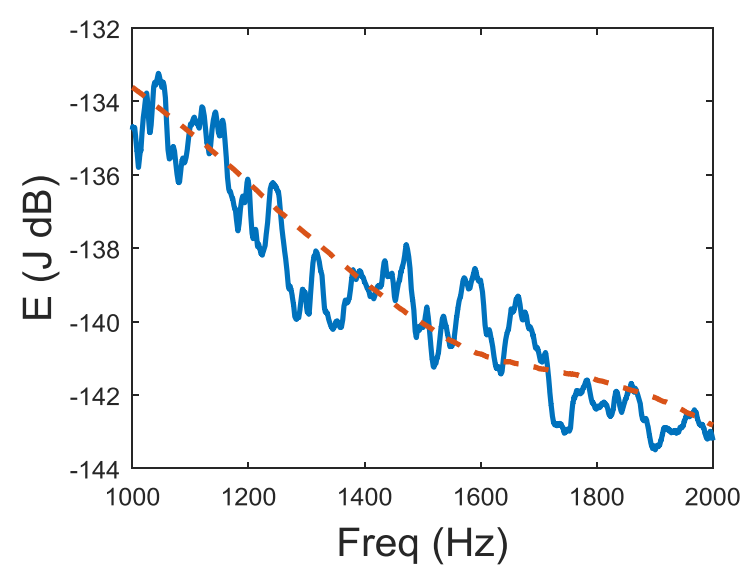

(b)

Figure 11: Mean energies in the frequency range $1000-2000 \mathrm{~Hz}$ predicted by SEA (dashed) compared with experiment (solid) for plate one: (a) transient mean and (b) steady-state mean.

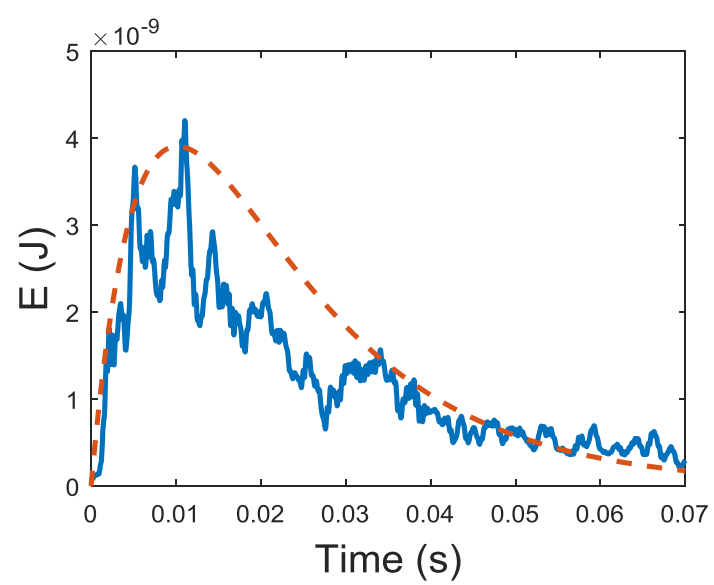

(a)

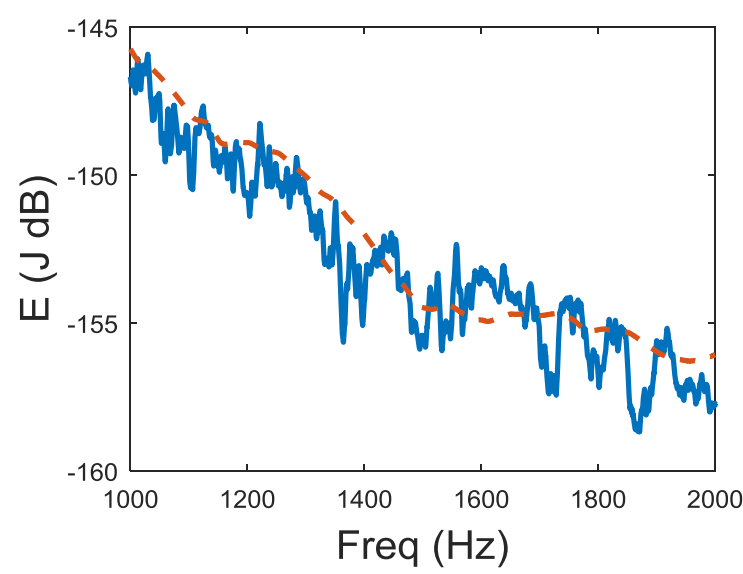

(b)

Figure 12: Mean energies in the frequency range $1000-2000 \mathrm{~Hz}$ predicted by SEA (dashed) compared with experiment (solid) for plate two: (a) transient mean and (b) steady-state mean. 
Additionally, the TSEA results in the frequency range $2000-3000 \mathrm{~Hz}$ are displayed in Figures 13 and 14 where greater similarity between experiment and theory is observed because the steady-state results are more similar. Statistical Energy Analysis can reasonably be applied to both of these frequency ranges. However, the difference in the comparison to the benchmark results between the two frequency ranges highlights how the accuracy of the transient results is dictated by the corresponding steady-state ones.

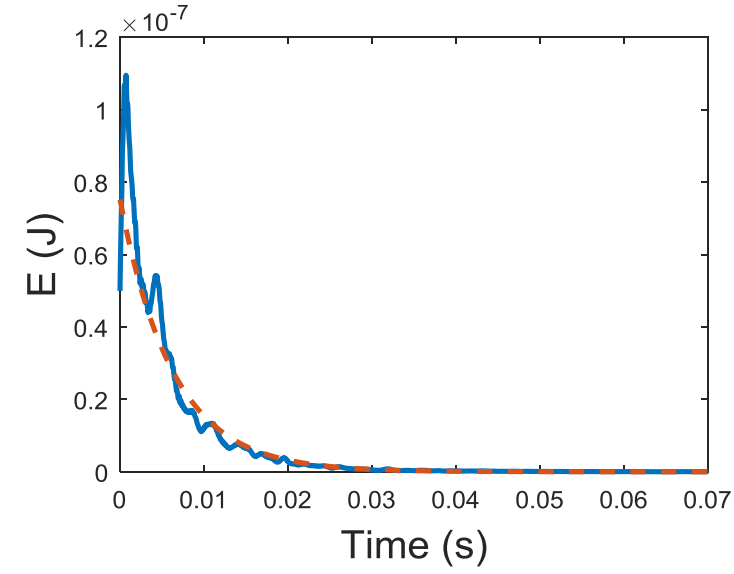

(a)

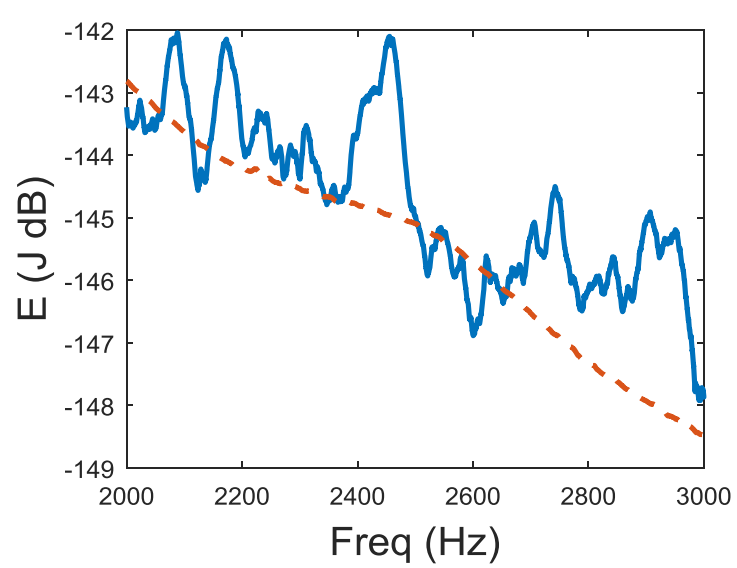

(b)

Figure 13: Mean energies in the frequency range $2000-3000 \mathrm{~Hz}$ predicted by SEA (dashed) compared with experiment (solid) for plate one: (a) transient mean and (b) steady-state mean.

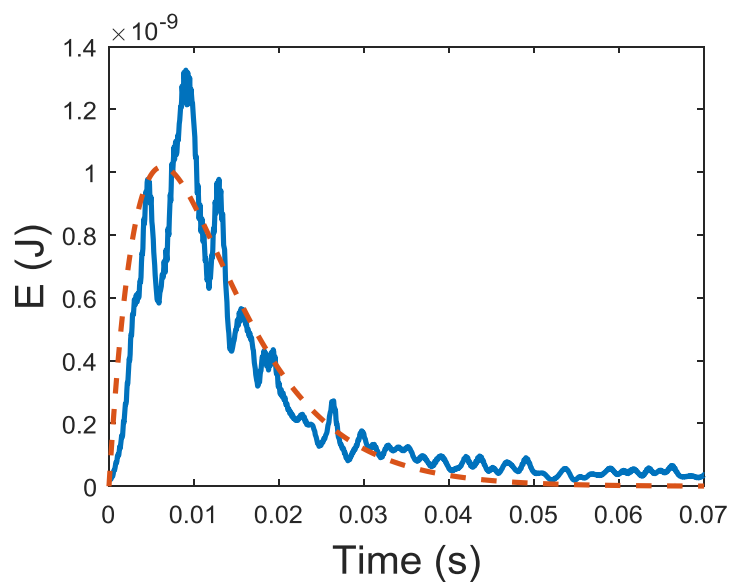

(a)

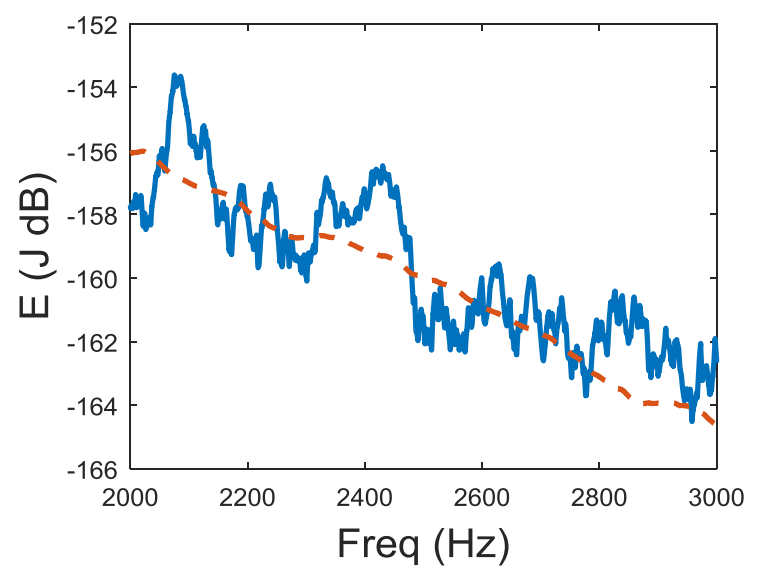

(b)

Figure 14: Mean energies in the frequency range $2000-3000 \mathrm{~Hz}$ predicted by SEA (dashed) compared with experiment (solid) for plate two: (a) transient mean and (b) steady-state mean.

Whilst the experimental results demonstrate good agreement with the theory, they also show differences; in particular they oscillate around a mean value and contain a spike immediately after the impulse in plate one. Both of these are due to estimating the total plate energy from only five 
accelerometers. The spike after the impulse occurs because the total energy has been calculated as two times the kinetic energy, but immediately after an impulse all of the energy is kinetic hence there is an overprediction until the assumption that on average across the ensemble and averaged over the accelerometers, the total energy is equal to two times the kinetic energy.

The issue of the oscillatory mean response is due to assuming that taking acceleration measurements at a finite number of points is sufficient to reliably calculate the energy of the entire plate. This can be illustrated by investigating the energy from individual realisations, three of which are plotted in Figure 15. As well as demonstrating the level of randomisation achieved by randomly distributing added masses, the energy time histories show that the energy in each plate oscillates. This is not due to energy flow between the plates since the energy levels in each plate are very different, so must be caused by spatial fluctuations in energy whereby at any one time, the kinetic energy at the five accelerometer points could be higher or lower than the plate average.

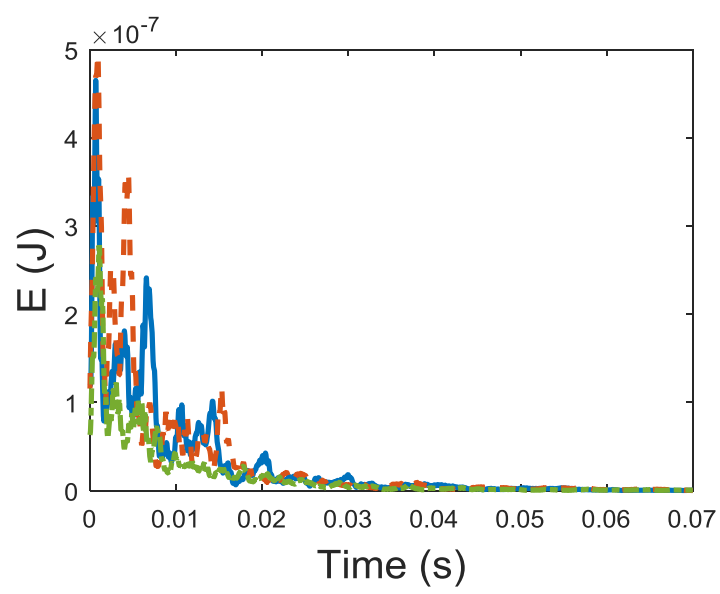

(a)

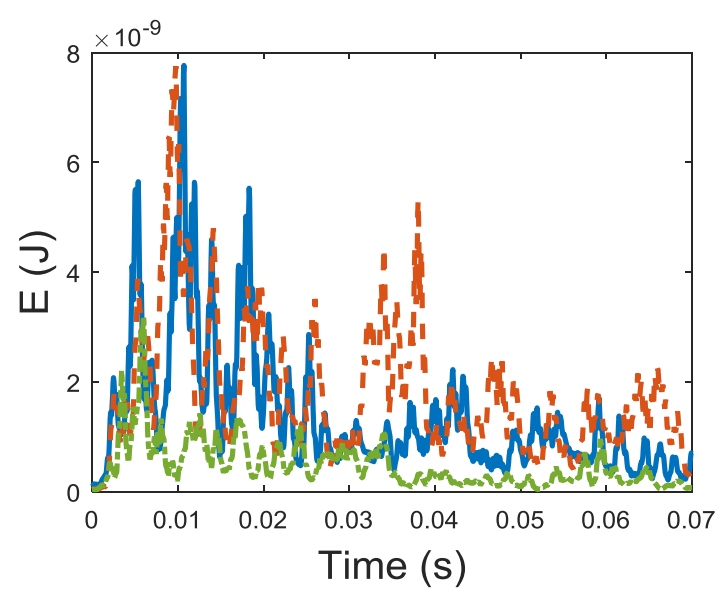

(b)

Figure 15: Experimental plate energies of three realisations in the frequency range $1000-2000 \mathrm{~Hz}$, (a) plate one and (b) plate two.

This effect can be investigated using the finite element analysis of the system in Figure 1, but where the total energy is calculated by observing the velocity at a finite number of points in order to mimic the experimental approach. Figure 16 shows the energy of three realisations where only five points are observed on each plate and a similar phenomenon to the experiment in Figure 15 is displayed. Figure 17 compares the average energy using five realisations with five observation points against using 100 realisations with five observation points or five realisations with 100 observation points. As with the experimental results, a moving mean filter has been used to smooth the results and where 
less averaging occurs, the mean response is more oscillatory. The effect of improved averaging either from more observation points or more realisations shows that the oscillations in the mean plate energies can be reduced.

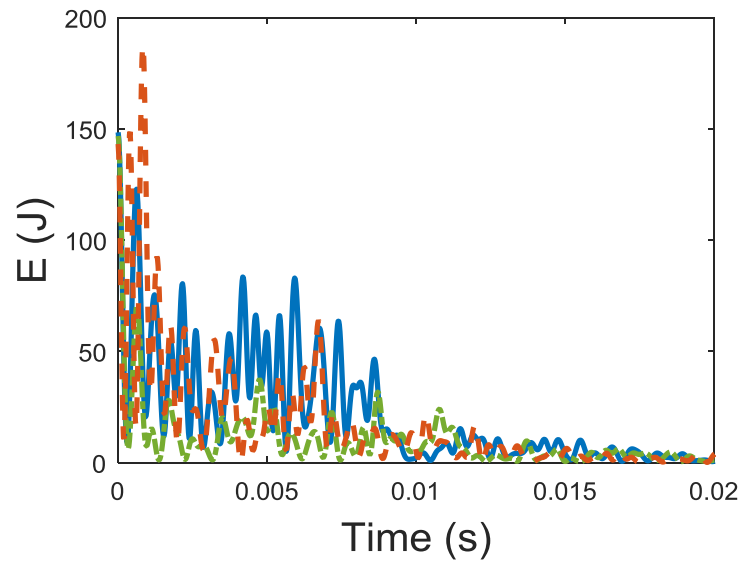

(a)

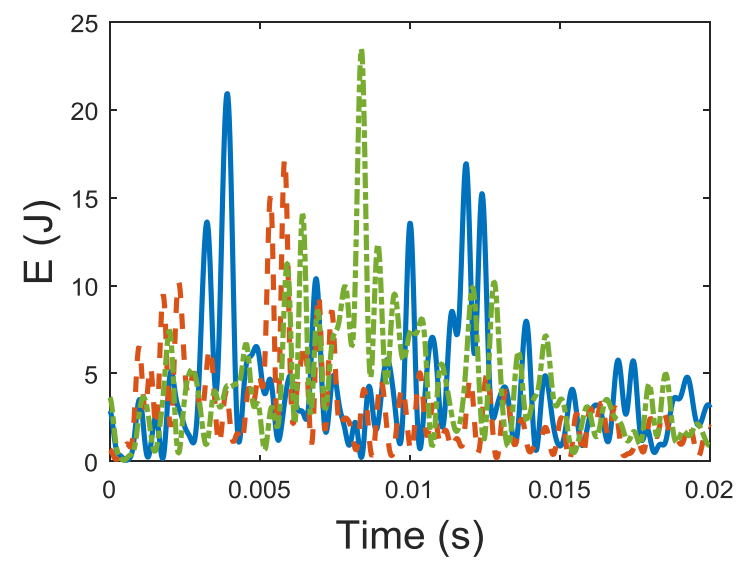

(b)

Figure 16: Plate energies from finite element results of three realisations of two plate system of Figure 1 where energy is calculated from observing the velocity at five points. (a) Plate one and (b) plate two.

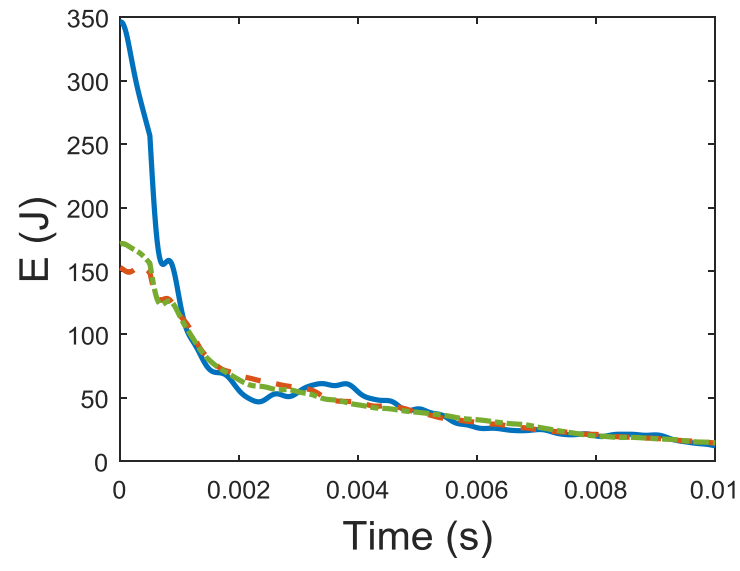

(a)

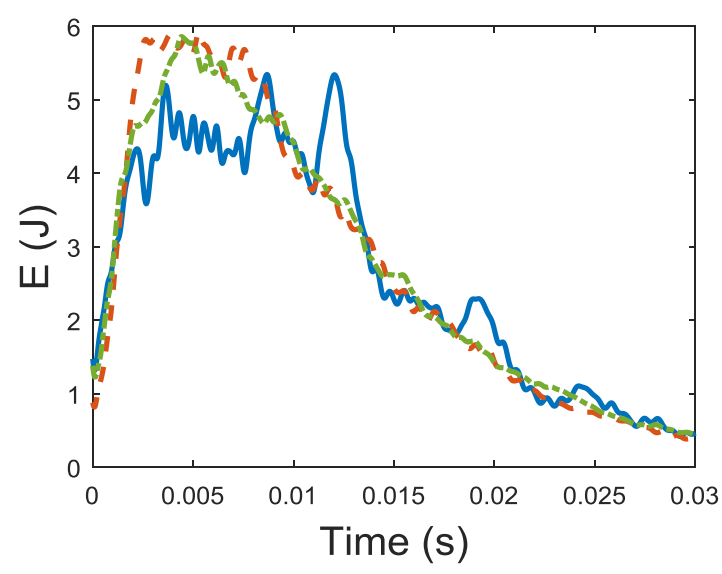

(b)

Figure 17: Mean plate energies from finite element results of two plate system of Figure 1 where energy is calculated from five realisations and five observation points (solid), five realisations and 100 observation points (dashed) and 100 realisations and five observation points (dash-dot). (a) Plate one and (b) plate two. 


\section{Conclusions}

A general method for applying Transient Statistical Energy Analysis has been presented that accounts for the time and frequency dependence of the subsystem energies and provides a suitable initial condition for the energy in the forced subsystem. The Priestley description of a non-stationary vibration is adapted and applied to an ensemble of random systems to describe the time evolution of the subsystem energies. The solution method for the TSEA equation is described and Parseval's theorem was employed to generate initial conditions for the subsystem energies under impulsive excitation that provide the correct area under the transient energy curves provided SEA for the system under steady-state loading is correct. Consequently, after an impulsive load at a point, the initial energy in the forced subsystem can be calculated as $8 \pi$ times the power input from steady-state harmonic point excitation.

The TSEA method has been validated by comparison to both finite element simulations and experimental investigation of impulsively excited plates. Strong agreement between the TSEA predictions and the benchmark methods is observed, with the initial conditions, rise times, peak energies and decay rates all being well predicted. This suggests that use of the TSEA equation is valid and the steady-state loss factors are sufficient to provide good accuracy. Reliance of the transient results on the corresponding steady-state results was demonstrated, meaning that the accuracy of the theory is limited by steady-state SEA theory which has been investigated extensively for many years.

In its current form, TSEA predicts only the mean energy evolution of each subsystem of an ensemble of random systems. However, as observed in Figure 15, the energy evolution of each realisation of the ensemble varies significantly around the mean. As such, an extension to the TSEA theory to quantify the variability of the energy evolution across the ensemble is also important and is therefore the focus of an accompanying paper.

\section{Acknowledgements}

The authors would like to thank Mitsubishi Heavy Industries for funding this research. 


\section{Appendix: Use of the Priestley description for random systems}

The Priestley representation of a function, Eq. (8), was introduced by Priestley to describe a nonstationary random process. In the present application, the loading on the system is deterministic, and hence the system response does not form a random process in the sense of Priestley; instead, the randomness arises from the random properties of the system. To examine the use of the Priestley formalism in the present context, consider initially a stationary diffuse wavefield representation of a response variable $v(t)$ so that

$$
v(t)=\operatorname{Re} \int_{0}^{\infty} \int_{0}^{2 \pi} A(\omega, \theta) \exp \{-\mathrm{i} \mathbf{k}(\omega, \theta) \cdot \mathbf{r}+\mathrm{i} \omega t\} \mathrm{d} \omega \mathrm{d} \theta
$$

where $A(\omega, \theta)$ is the amplitude of the wave with frequency $\omega$ and heading $\theta$ and Re represents the real part. The square of the response is given by

$$
\begin{aligned}
& v^{2}(t)=\frac{1}{2} \operatorname{Re} \int_{0}^{\infty} \int_{0}^{\infty} \int_{0}^{2 \pi} \int_{0}^{2 \pi} A\left(\omega_{1}, \theta_{1}\right) A^{*}\left(\omega_{2}, \theta_{2}\right) \mathrm{e}^{\left\{-\mathrm{ik}\left(\omega_{1}, \theta_{1}\right) \cdot \mathbf{r}+\mathbf{i k}\left(\omega_{2}, \theta_{2}\right) \cdot \mathbf{r}+\mathrm{i} \omega_{1} t-\mathrm{i} \omega_{2} t\right\}} \mathrm{d} \omega_{1} \mathrm{~d} \theta_{1} \mathrm{~d} \omega_{2} \mathrm{~d} \theta_{2} \\
&+\frac{1}{2} \operatorname{Re} \int_{0}^{\infty} \int_{0}^{\infty} \int_{0}^{2 \pi} \int_{0}^{2 \pi} A\left(\omega_{1}, \theta_{1}\right) A\left(\omega_{2}, \theta_{2}\right) \mathrm{e}^{\left\{-\mathbf{i k}\left(\omega_{1}, \theta_{1}\right) \cdot \mathbf{r}-\mathrm{ik}\left(\omega_{2}, \theta_{2}\right) \cdot \mathbf{r}+\mathrm{i} \omega_{1} t+\mathrm{i} \omega_{2} t\right\}} \mathrm{d} \omega_{1} \mathrm{~d} \theta_{1} \mathrm{~d} \omega_{2} \mathrm{~d} \theta_{2}
\end{aligned}
$$

and the ensemble mean of this quantity can be derived by considering the statistical properties of the wave amplitudes. Taking these amplitudes to be statistically independent complex variables (i.e. assuming a diffuse field), it follows that

$$
\begin{gathered}
\mathrm{E}\left[A\left(\omega_{1}, \theta_{1}\right) A^{*}\left(\omega_{2}, \theta_{2}\right)\right]=f(\omega, \theta) \delta\left(\omega_{1}-\omega_{2}\right) \delta\left(\theta_{1}-\theta_{2}\right), \\
\mathrm{E}\left[A\left(\omega_{1}, \theta_{1}\right) A\left(\omega_{2}, \theta_{2}\right)\right]=0,
\end{gathered}
$$

and the ensemble mean squared value of the response is given by

$$
\mathrm{E}\left[v^{2}(t)\right]=\int_{0}^{\infty} \int_{0}^{2 \pi} f(\omega, \theta) \mathrm{d} \omega \mathrm{d} \theta=\int_{0}^{\infty} g(\omega) \mathrm{d} \omega, \quad g(\omega)=\int_{0}^{2 \pi} f(\omega, \theta) \mathrm{d} \theta
$$

It follows from equation (A.1) that the response can also be written in the form

$$
\begin{gathered}
v(t)=\operatorname{Re} \int_{0}^{\infty} v(\omega) \mathrm{e}^{\mathrm{i} \omega t} \mathrm{~d} \omega, \\
v(\omega)=\int_{0}^{2 \pi} A(\omega, \theta) \exp \{-\mathrm{ik}(\omega, \theta) \cdot \mathbf{r}\} \mathrm{d} \theta .
\end{gathered}
$$

The statistical properties of the function $v(\omega)$ can be deduced from equations (A.3), (A.4) and (A.8), so that, for example 


$$
\begin{gathered}
\mathrm{E}\left[v\left(\omega_{1}\right) v^{*}\left(\omega_{2}\right)\right]=\int_{0}^{2 \pi} \int_{0}^{2 \pi} \mathrm{E}\left[A\left(\omega_{1}, \theta_{1}\right) A^{*}\left(\omega_{2}, \theta_{2}\right)\right] \mathrm{e}^{\left\{-\mathrm{ik}\left(\omega_{1}, \theta_{1}\right) \cdot \mathbf{r}+\mathrm{ik}\left(\omega_{2}, \theta_{2}\right) \cdot \mathbf{r}\right\}} \mathrm{d} \theta_{1} \mathrm{~d} \theta_{2}, \\
\Rightarrow \quad \mathrm{E}\left[v\left(\omega_{1}\right) v^{*}\left(\omega_{2}\right)\right]=g(\omega) \delta\left(\omega_{1}-\omega_{2}\right) .
\end{gathered}
$$

It follows that equation (A.7) can be expressed in the alternative way

$$
v(t)=\int_{-\infty}^{\infty} \mathrm{e}^{\mathrm{i} \omega t} \mathrm{~d} S(\omega)
$$

where

$$
\mathrm{d} S(-\omega)=\mathrm{d} S^{*}(\omega), \quad \mathrm{E}\left[\mathrm{d} S\left(\omega_{1}\right) \mathrm{d} S^{*}\left(\omega_{2}\right)\right]=\frac{1}{2} g\left(\omega_{1}\right) \delta\left(\omega_{1}-\omega_{2}\right) \mathrm{d} \omega_{1} \mathrm{~d} \omega_{2}
$$

These results are identical to the corresponding description of a stationary random process, the only difference being that the ensemble average is taken here over an ensemble of random structures, rather than over an ensemble of random processes. Equation (A.11) is readily extended to transient (non-stationary) case by using the same approach as Priestley, which yields

$$
v(t)=\int_{-\infty}^{\infty} V(\omega, t) \mathrm{e}^{\mathrm{i} \omega t} \mathrm{~d} S(\omega)
$$

This result forms the basis of the analysis contained in this paper. 


\section{References}

[1] R.H. Lyon, R.G. DeJong, Theory and application of statistical energy analysis, ButterworthHeinemann, Boston, 1995.

[2] J.E. Manning, K. Lee, Predicting mechanical shock transmission, Shock Vib. Bull. 37 (1968) 6570.

[3] R. Pinnington, D. Lednik, Transient statistical energy analysis of an impulsively excited two oscillator system, J. Sound Vib. 189 (1996) 249-264. doi:10.1006/jsvi.1996.0018.

[4] R.J. Pinnington, D. Lednik, Transient energy flow between two coupled beams, J. Sound Vib. 189 (1996) 265-287. doi:http://dx.doi.org/10.1006/jsvi.1996.0019.

[5] M.L. Lai, A. Soom, Prediction of transient vibration envelopes using statistical energy analysis techniques, J. Vib. Acoust. 112 (1990) 127-137. doi:10.1115/1.2930088.

[6] M.L. Lai, A. Soom, Statistical energy analysis for the time-integrated transient response of vibrating systems, J. Vib. Acoust. 112 (1990) 206-213. doi:10.1115/1.2930114.

[7] M. Robinson, C. Hopkins, Prediction of maximum time-weighted sound and vibration levels using transient statistical energy analysis. Part 2 : Experimental validation, Acta Acust. United with Acust. 100 (2014) 57-66. doi:10.3813/AAA.918686.

[8] M.B. Priestley, Power spectral analysis of non-stationary random processes, J. Sound Vib. 6 (1967) 86-97. doi:10.1016/0022-460X(67)90160-5.

[9] R.S. Langley, On quasi-stationary approximations to non-stationary random vibration, J. Sound Vib. 113 (1987) 365-375. doi:10.1016/S0022-460X(87)80222-5.

[10] F.S. Sui, M.N. Ichchou, L. Jezequel, Prediction of vibroacoustics energy using a discretized transient local energy approach and comparison with TSEA, J. Sound Vib. 251 (2002) 163180. doi:10.1006/jsvi.2001.3786.

[11] M. Robinson, C. Hopkins, Prediction of maximum time-weighted sound and vibration levels using transient statistical energy analysis. Part 1: Theory and numerical implementation, Acta Acust. United with Acust. 100 (2014) 46-56. doi:10.3813/AAA.918685.

[12] C. Hopkins, M. Robinson, On the evaluation of decay curves to determine structural reverberation times for building elements, Acta Acust. United with Acust. 99 (2013) 226-244. doi:10.3813/AAA.918606. 
[13] B.Y. Mao, S.L. Xie, M.L. Xu, X.N. Zhang, G.H. Zhang, Simulated and experimental studies on identification of impact load with the transient statistical energy analysis method, Mech. Syst. Signal Process. 46 (2014) 307-324. doi:10.1016/j.ymssp.2014.01.015.

[14] M. Wright, R. Weaver, New directions in linear acoustics and vibration: Quantum chaos, random matrix theory, and complexity, Cambridge University Press, New York, 2010. doi:10.1017/CBO9780511781520.

[15] R.L. Weaver, O.I. Lobkis, Anderson localization in coupled reverberation rooms, J. Sound Vib. 231 (2000) 1111-1134. doi:10.1006/jsvi.1999.2725.

[16] B.R. Mace, Statistical energy analysis: Coupling loss factors, indirect coupling and system modes, J. Sound Vib. 279 (2005) 141-170. doi:10.1016/j.jsv.2003.10.040.

[17] O. Guasch, C. García, Numerical local time stepping solutions for transient statistical energy analysis, J. Vib. Acoust. 136 (2014) 064502. doi:10.1115/1.4028454.

[18] L. Cremer, M. Heckl, B.A.T. Petersson, Structure-borne sound: Structural vibrations and sound radiation at audio frequencies, 2005. doi:10.1007/b137728. 